\title{
Coherent Anti-Stokes Raman Holography for Chemically Selective Single-Shot Nonscanning 3D Imaging
}

\author{
Kebin Shi, ${ }^{1}$ Haifeng Li, ${ }^{1}$ Qian Xu, ${ }^{1}$ Demetri Psaltis, ${ }^{2}$ and Zhiwen $\mathrm{Liu}^{1, *}$ \\ ${ }^{1}$ Department of Electrical Engineering, the Pennsylvania State University, University Park, Pennsylvania 16802, USA \\ ${ }^{2}$ School of Engineering, Ecole Polytechnique Fédérale de Lausanne, Lausanne, Switzerland
}

(Received 20 November 2009; published 4 March 2010)

\begin{abstract}
We propose and develop a new way for nonscanning coherent anti-Stokes Raman scattering (CARS) imaging by using CARS holography, which can capture, at a single shot, both the amplitude and the phase of a coherent Raman image. It hence allows for chemically selective three-dimensional imaging at a laser pulse duration limited speed, which is not possible to achieve by using any of the existing laser scanning based Raman imaging techniques. We have demonstrated single-shot (pulse duration: $\sim 5 \mathrm{~ns}$ ) holographic CARS imaging of microspheres. Our results show that coherent anti-Stokes Raman holography can open a new path for nonscanning label-free imaging.
\end{abstract}

DOI: 10.1103/PhysRevLett.104.093902

Coherent anti-Stokes Raman scattering (CARS) microscopy [1-6] has emerged as a promising imaging technique. It directly utilizes molecular vibrational response as a contrast mechanism and is therefore label-free. Equally important is the fact that CARS can have significantly improved sensitivity over spontaneous Raman scattering. As a result, CARS microscopy including both the laserscanning [1-3] and wide field [4-6] modalities has been extensively investigated recently. Despite the many unique advantages of CARS microscopy, scanning is required to perform three-dimensional (3D) CARS imaging. To overcome the limitation, here we explore coherent anti-Stokes Raman holography (CARS holography) as a new way for nonscanning 3D CARS imaging. In contrast to microscopy, holography is known to have unique 3D imaging capability. Holography has been applied to fluorescence $[7,8]$ and second harmonic $[9,10]$ imaging. However, unlike CARS holography these holographic imaging techniques usually require the use of fluorescent or second harmonic nanocrystal markers. As a related technique, interferometry has been previously applied to CARS imaging. For instance, CARS spectral interferometry [11] was utilized to obtain the real and imaginary parts of the relevant third order nonlinear susceptibility for suppression of nonresonant background. CARS optical coherence tomography [12] and phase contrast CARS imaging [13,14] have also been previously demonstrated. These prior studies were primarily concerned with interferometric detection of CARS signals generated from diffraction-limited spots and scanning is needed to obtain an image. CARS holography, on the other hand, can capture both the amplitude and the phase of a wide-field CARS image. In essence, it has the unique capabilities of both CARS and holography.

Figure 1(a) illustrates the basic principle of CARS holographic imaging. Briefly, a pump (also used as a probe) beam and a tunable Stokes beam with respective angular frequencies $\omega_{p}$ and $\omega_{s}$ interact with a sample to produce a four wave mixing (FWM) signal, or resonant CARS signal
PACS numbers: 42.65.Dr, 42.30.- d, 42.40.-i

if the frequency difference between the pump and the Stokes $\left(\omega_{p}-\omega_{s}\right)$ matches the molecular vibrational frequency of the sample. A reference beam $R e^{-j\left(2 \omega_{p}-\omega_{s}\right) t}$ with angular frequency $2 \omega_{p}-\omega_{s}$ then interferes with the generated CARS/FWM image $S e^{-j\left(2 \omega_{p}-\omega_{s}\right) t}$ to record a digital CARS/FWM hologram $|R+S|^{2}$. The complex CARS field $S$ can then be reconstructed by the following procedure [15]: first the two-dimensional (2D) Fourier transform of the recorded digital hologram is calculated; then one side band (e.g., corresponding to the term $R^{*} S$ ) is selected through digital filtering; finally, the complex CARS image field can be obtained through the inverse 2D Fourier transform of the selected band. Three-dimensional CARS imaging can then be achieved by digitally propagating the reconstructed complex CARS field. The digital propagation is realized by first taking the 2D Fourier transform of the complex field, then multiplying it by the transfer function [16] $e^{j k_{z} L}$ [where $k_{z}=\sqrt{(2 \pi / \lambda)^{2}-k_{x}^{2}-k_{y}^{2}}, \lambda$ is the wavelength in the medium, $\left(k_{x}, k_{y}, k_{z}\right)$ is the wave vector, and $|L|$ is the propagation distance], and finally taking the inverse Fourier transform.

The schematic diagram of our experimental system is shown in Fig. 1(b). The output from a nanosecond pulsed laser at fundamental angular frequency $\omega_{p}$ (Continuum Surelite III, $\lambda_{1}=1064 \mathrm{~nm}$, linewidth: $0.005 \mathrm{~cm}^{-1}$, repetition rate: $10 \mathrm{~Hz}$, pulse duration $\sim 5 \mathrm{~ns}$, injection seeded) is used as the pump for generating the CARS/FWM signal. The frequency-doubled laser beam at $2 \omega_{p}\left(\lambda_{2}=532 \mathrm{~nm}\right)$ pumps a tunable type II optical parametric oscillator (Photop Technologies, OPO BBO-2B) to produce an idler beam at $\omega_{s}$ which is utilized as the Stokes beam, and a signal at $2 \omega_{p}-\omega_{s}$ which is used as the reference beam for recording CARS holograms. Note that the frequency of the reference beam automatically matches that of the CARS/ FWM signal as the Stokes (or idler) wavelength is tuned. The pump and Stokes beams are focused onto a sample (lens focal length: $750 \mathrm{~mm}$ and $150 \mathrm{~mm}$, respectively), and 

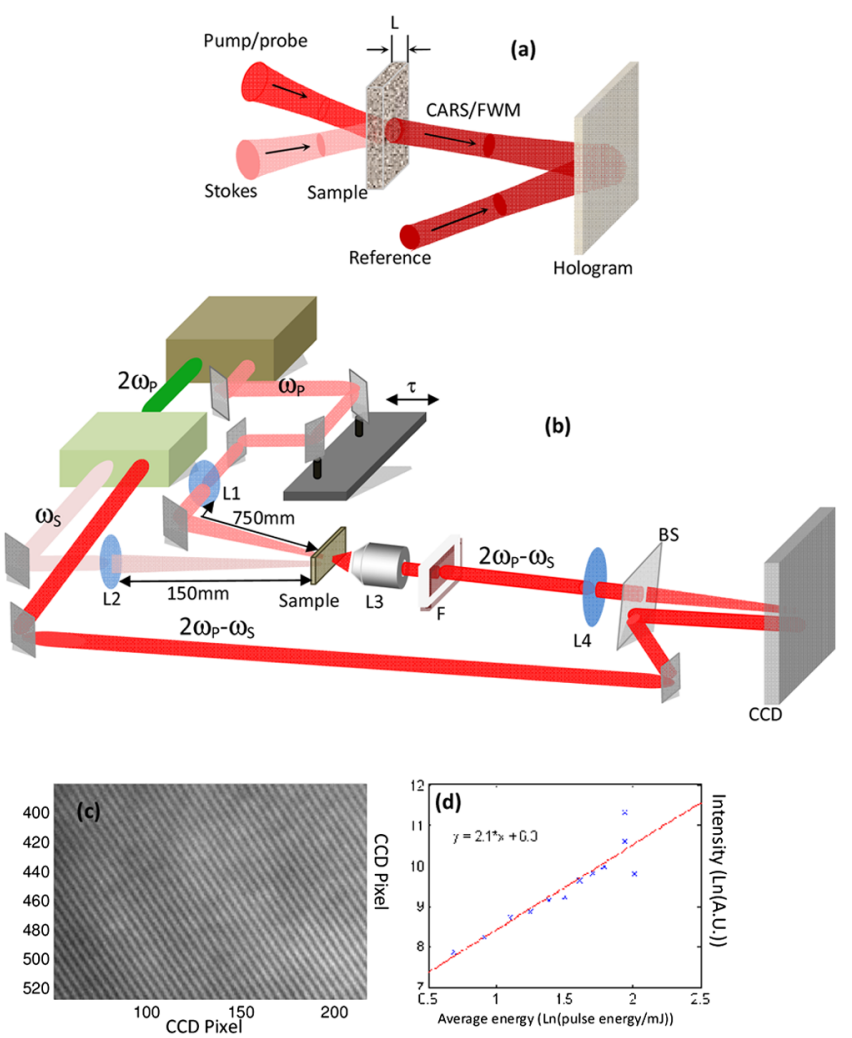

FIG. 1 (color online). (a) Schematic diagram illustrating the principle of coherent anti-Stokes Raman holography, (b) Schematic diagram of the experimental setup. L1: singlet lens, focal length $750 \mathrm{~mm}$, L2: singlet lens, focal length $150 \mathrm{~mm}$, L3: long working distance objective lens, focal length $10 \mathrm{~mm}$, L4: singlet lens, focal length $300 \mathrm{~mm}$ or $500 \mathrm{~mm}$, F: band-pass filter, BS: pellicle beam splitter. (c) A typical hologram recorded on a CCD camera by interfering a single pair of reference pulse and signal pulse generated by FWM in index oil. (d) Dependence of CARS signal on the power of the pump beam in logarithmic scale.

are spatially overlapped at the sample plane with an angle of about 20 degrees between them. Optimal temporal overlapping between the two beams can be obtained by adjusting the delay line in the pump beam path. A CARS/FWM image with a field of view diameter of about $120 \mu \mathrm{m}$ can be obtained. A small piece of Teflon is used to block the transmitted pump and Stokes beams. The generated CARS/ FWM image is then magnified by an imaging system consisting of an objective (numerical aperture: 0.42 , focal length: $10 \mathrm{~mm}$ ) and a lens, filtered by a band-pass filter (ChromaD800/30), and detected by a CCD camera (Apogee 32ME). To record a hologram, the reference beam first passes through a half wave plate to adjust its polarization. It is subsequently attenuated and spatially filtered before combining with the CARS/FWM beam by using a pellicle beam splitter (reflectivity and transmittance $\sim 8 \%$ and $92 \%$, respectively) placed in front of the CCD camera. A delay line in the reference beam path (not shown in Fig. 1) is adjusted to ensure optimal temporal overlapping between the CARS/FWM beam and the reference beam. The two beams then interfere on the CCD camera to record a CARS/FWM hologram. Figure 1(c) shows a typical hologram recorded by interfering a single pair of reference pulse and FWM signal pulse generated from index oil. Interference fringes can be clearly observed. The period of the fringes is about $27 \mu \mathrm{m}$, consistent with an angle of approximately 1.7 degrees between the reference and the CARS/FWM beams.

We verified the quadratic dependence of the CARS signal on the power of the pump beam. To this end, we measured the CARS signal generated from a polystyrene microsphere (Duke Scientific, nominal diameter: $10 \mu \mathrm{m}$ ). The wavelength of the Stokes beam was tuned to resonantly excite molecular vibration (at around $3060 \mathrm{~cm}^{-1}$ [17]) in polystyrene sphere. The average energy of the pump pulses was varied from $2 \mathrm{~mJ}$ to $7.5 \mathrm{~mJ}$ with an increment of $0.5 \mathrm{~mJ}$ each time while that of the Stokes pulses was fixed at about $4 \mathrm{~mJ}$. All the pulse energy measurements were performed right in front of the sample. The generated CARS signal strength was obtained by summing up the pixel values of the CARS signal captured on the CCD camera. An exposure time of 30 seconds was used during the measurement. Figure 1(d) shows the dependence of the measured CARS signal strength from a polystyrene sphere on the average pulse energy of the pump beam in logarithmic scale. The measured data can be fitted by a linear curve with a slope of 2.1, indicating a quadratic relationship as expected. Deviation of the measured data from an ideal quadratic relationship is primarily caused by the shot-to-shot fluctuation of the optical parametric oscillator and the nanosecond laser and the limited accuracy in pulse energy measurement.

Like CARS microscopy, a unique advantage of CARS holography is its chemical selective imaging capability. To demonstrate this, a sample was prepared by sandwiching a PMMA(poly(methylmethacrylate)) microsphere (Bangs Lab., nominal diameter: $15 \mu \mathrm{m}$ ) and two polystyrene microspheres (Duke Scientific, nominal diameter: $10 \mu \mathrm{m}$ ) between a pair of microscope cover glasses (VWR No.1 cover glass). The microspheres were all immobilized on one of the cover glasses by using UV curable optical adhesive (Norland Optical Adhesive 60) and immersed in water. The edges of the cover glasses were sealed with silicone vacuum sealant. Figures 2(a) and 2(b) are the recorded CARS holograms when the Stokes wavelength was tuned to match the molecular vibrational frequencies of polystyrene and PMMA, respectively. An exposure time of $90 \mathrm{~ms}$ was used to ensure single-shot recording as the repetition rate of the laser system was $10 \mathrm{~Hz}$. When the Stokes wavelength was tuned to match the polystyrene resonance (corresponding to the Raman resonance at $3060 \mathrm{~cm}^{-1}$ [17]), a strong CARS signal from the two polystyrene spheres can be observed in the recorded hologram [Fig. 2(a)]. Only a weak nonresonant FWM signal was generated from the PMMA sphere in the middle. On the other hand, when the Stokes wavelength was tuned to match the PMMA resonance (corresponding to the Raman 
Polystyrene microspheres

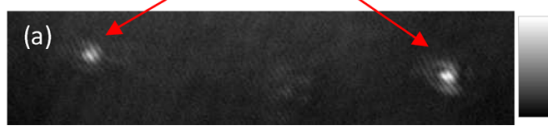

(c)
PMMA microsphere

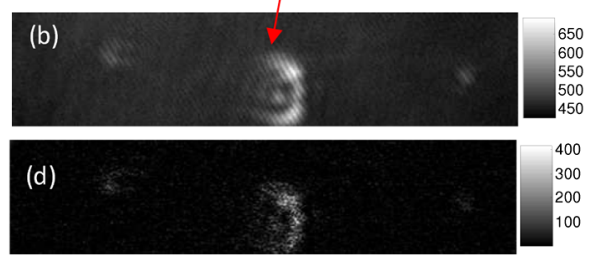

FIG. 2 (color online). Chemical selective CARS holographic imaging. (a) single-shot CARS hologram recorded at polystyrene resonance wavelength; (b) single-shot hologram recorded at PMMA resonance wavelength; (c) reconstructed CARS intensity image from the hologram shown in (a); (d) reconstructed CARS intensity image from the hologram shown in (b).

resonance at $2959 \mathrm{~cm}^{-1}$ [17]), the situation was reversed. Strong CARS signal from the PMMA microsphere and weak nonresonant FWM signal from the polystyrene microspheres were recorded in the hologram as shown in Fig. 2(b). This clearly shows the unique chemical selective imaging capability of CARS holography. The intensity of digitally reconstructed CARS images corresponding to the holograms shown in Figs. 2(a) and 2(b) are shown in (c) and $(d)$, respectively.

One of the most important advantages of CARS holography is that it captures both the amplitude and the phase of a CARS image and can therefore have 3D imaging capability. Let us consider a sample with a uniform refractive index and a relevant third order nonlinear susceptibility $\chi^{(3)}(x, y, z)$. The generated anti-Stokes field satisfies the following equation [18],

$$
\left[\nabla^{2}+\frac{\omega^{2}}{c^{2}} n^{2}\right] E_{a s}=-\frac{4 \pi \omega^{2}}{c^{2}} \chi^{(3)}(x, y, z) E_{p}^{2} E_{s}^{*},
$$

where $\omega$ is the angular frequency of the CARS signal, $c$ is the speed of light in vacuum, $E_{p}$ and $E_{s}$ are the pump and Stokes fields. For simplicity, we use the scalar formalism and assume that the pump and Stokes are undepleted plane waves, i.e., $E_{p}=A_{p} e^{j \vec{k}_{p} \cdot \vec{r}}$ and $E_{s}=A_{s} e^{j \vec{k}_{s} \cdot \vec{r}}$ respectively, where $A_{p}$ and $A_{s}$ are the complex field amplitudes, $\vec{k}_{p}$ and $\vec{k}_{s}$ are the wave vectors. By applying the slowly varying envelop approximation [18], we obtain the output antiStokes field

$$
\begin{aligned}
E_{a s}(x, y, L)= & -\frac{4 \pi \omega^{2}}{c^{2}} A_{p}^{2} A_{s}^{*} e^{j k_{a s} L} \\
& \times \int_{0}^{L} d z e^{j \Delta k z}\left[\chi^{(3)}(x, y, z) * \frac{k_{a s}}{j 2 \pi(L-z)}\right. \\
& \left.\times e^{j\left(k_{a s} / 2(L-z)\right)\left(x^{2}+y^{2}\right)}\right]
\end{aligned}
$$

where $L$ is the sample thickness, $k_{a s}$ is the angular wave number of the generated anti-Stokes beam which propagates paraxially along the $z$ axis, $\Delta k=2 k_{p z}-k_{s z}-k_{a s}$, and "*" denotes two-dimensional (i.e., in $x$ and $y$ ) convolution. Note that the convolution kernel is actually the Fresnel diffraction kernel [16] and the anti-Stokes field is proportional to the superposition of "diffracted" $\chi^{(3)}(x, y, z)$ at the output plane. Three-dimensional CARS imaging can therefore be realized by digital propagation of the complex output anti-Stokes field which can be captured holographically. Other than a background term due to contributions from out-of-focus planes, the back-propagated field at $z$ is approximately proportional to $\chi^{(3)}(x, y, z)$.

We experimentally investigated holographic CARS imaging of multiple polystyrene microspheres (nominal diameter: $10 \mu \mathrm{m}$ ) suspended in water. The sample was similarly prepared by sandwiching polystyrene microspheres (dispersed in water) between a pair of microscope cover glasses with the edges sealed by silicone vacuum sealant. In the experiment, the energies of the pump and Stokes pulses were $8 \mathrm{~mJ}$ and $4 \mathrm{~mJ}$, respectively. The Stokes wavelength was tuned to match the polystyrene resonance (i.e., Raman resonance at $3060 \mathrm{~cm}^{-1}$ ). The CCD exposure time was set to $90 \mathrm{~ms}$ to ensure single-shot recording. Figure 3(a) shows a recorded CARS hologram. The reconstructed CARS intensity and phase image without diffraction compensation is shown in Figs. 3(b) and 3(c) respectively. Three out-of-focus microspheres can be seen in the figure. Since the CARS hologram captures the complex CARS image field, it can be propagated digitally to realize 3D imaging as aforementioned. Several field intensity distributions corresponding to different planes in the sample are shown in Fig. 3(d)-3(g). Note that the intensity distributions of the digitally propagated CARS fields in Figs. 3(d) and 3(f) have a much smaller spot size than that of the microspheres (diameter $\sim 10 \mu \mathrm{m}$ ), indicating that the microspheres can actually focus the generated CARS field. To confirm this lensing effect, we investigated CARS generation in a single 10 - $\mu \mathrm{m}$-diameter microsphere (refractive index: 1.59) immersed in water. Both the pump and Stokes beams are assumed to be undepleted and initially having Gaussian profiles with a propagation angle of $20^{\circ}$ between them. The scalar wave equations governing the propagation of the pump and Stokes beams and the generation of the anti-Stokes beam can be numerically solved by using the beam propagation method $[19,20]$. Our simulation results show that the generated antiStokes beam is converging after exiting the microsphere. Figure 3(h) shows the calculated intensity distribution of the generated anti-Stokes signal at a distance of $5 \mu \mathrm{m}$ away from the exit plane, which is in good qualitative agreement with the experimental observation. The above 

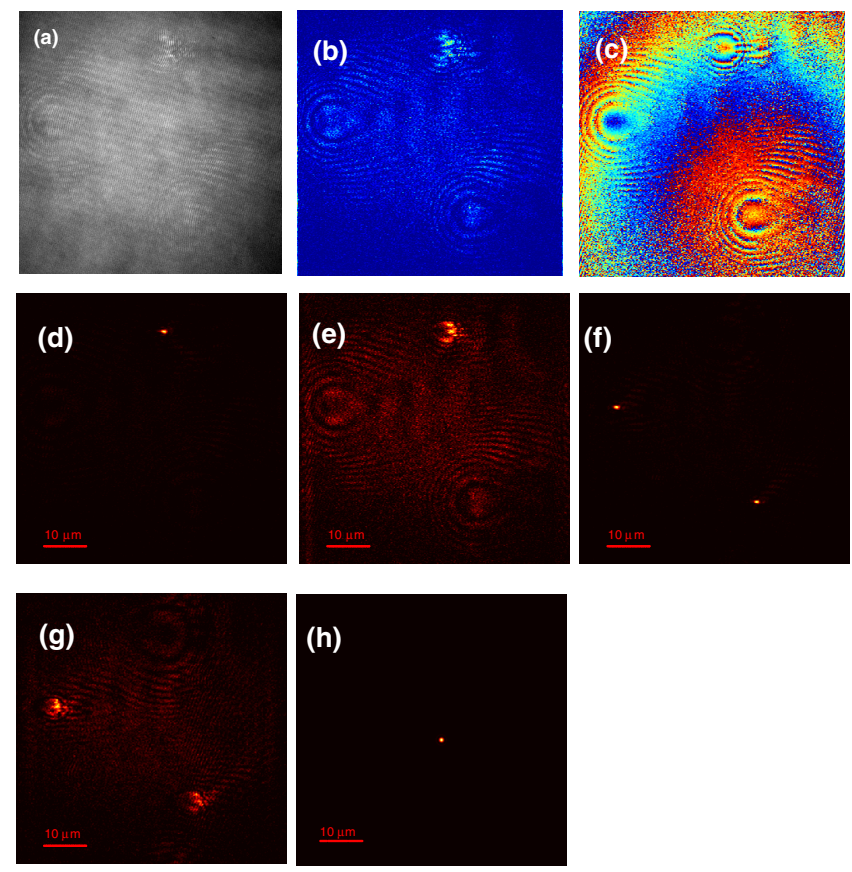

FIG. 3 (color online). Holographic CARS imaging of multiple polystyrene microspheres suspended in water. (a): recorded single-shot CARS hologram; (b): reconstructed CARS intensity image (without diffraction compensation); (c): reconstructed CARS phase image (without diffraction compensation); (d)(g): intensity distribution of digitally propagated CARS field at different planes; from (d) to (g): $z=7.92 \mu \mathrm{m}, 2.92 \mu \mathrm{m}$, $-15.6 \mu \mathrm{m},-20.6 \mu \mathrm{m}$, respectively; (h): calculated CARS intensity distribution of a single 10- $\mu$ m-diameter microsphere at a distance of $5 \mu \mathrm{m}$ away from the exit plane by using the beam propagation method; it is shown that the generated CARS field is focused by the microsphere to result in a much smaller spot size.

analysis suggests that in order to obtain the CARS images of the microspheres, we need to continue the digital propagation for about $5 \mu \mathrm{m}$ in addition until it reaches the exit plane of the microspheres. The results are shown in Figs. 3(e) and 3(g). Our results show that CARS images of polystyrene microspheres at different axial positions can all be digitally brought to focus. This indicates that CARS holographic imaging has a large depth of field and can therefore image a three-dimensional scene. We would like to point out that one will still need some prior knowledge of the object itself in order to choose a proper digital propagation distance, much in the same way as one requires experience to adjust the focus of a microscope in order to obtain a clear and meaningful image of a specimen. The advantage of CARS holography is that this analogue "focus adjusting" process is replaced by digital propagation and as a result a 3D scene can be captured in a single exposure and later on reconstructed by "digital focusing." It should be also noted that in general a specimen would not always focus the generated anti-Stokes field and therefore a proper digital propagation distance can be usually chosen easily.
In summary, we have demonstrated CARS holographic imaging, which combines the unique capabilities of both CARS and holography. By capturing both the amplitude and the phase of a complex CARS image field, CARS holography can have a large depth of field and therefore chemical-selectively image a three-dimensional object without scanning. Our results show that a CARS hologram can be recorded in a single shot, opening the possibility of three-dimensional imaging of fast dynamical phenomena at laser pulse-width limited speed. Finally, the presence of an intense reference beam (i.e., local oscillator) and the coherent homodyne detection nature of CARS holography can also potentially lead to shot noise limited detection sensitivity.

This work is supported by the National Science Foundation (DBI-0649866 and ECCS-0547475).

*To whom correspondence should be addressed. zliu@psu.edu

[1] M. D. Duncan, J. Reintjes, and T. J. Manuccia, Opt. Lett. 7, 350 (1982).

[2] A. Zumbusch, G. R. Holtom, and X. S. Xie, Phys. Rev. Lett. 82, 4142 (1999).

[3] J. X. Cheng and X.S. Xie, J. Phys. Chem. B 108, 827 (2004).

[4] C. Heinrich, S. Bernet, and M. Ritsch-Marte, Appl. Phys. Lett. 84, 816 (2004).

[5] I. Toytman, K. Cohn, T. Smith, D. Simanovskii, and D. Palanker, Opt. Lett. 32, 1941 (2007).

[6] C. Heinrich, S. Bernet, and M. Ritsch-Marte, New J. Phys. 8, 36 (2006).

[7] B. W. Schilling, T. C. Poon, G. Indebetouw, B. Storrie, K. Shinoda, Y. Suzuki, and M. H. Wu, Opt. Lett. 22, 1506 (1997).

[8] J. Rosen and G.Brooker, Nat. Photon. 2, 190 (2008).

[9] Y. Pu, M. Centurion, and D. Psaltis, Appl. Opt. 47, A103 (2008).

[10] C. L. Hsieh, R. Grange, Y. Pu, and D. Psaltis, Opt. Express 17, 2880 (2009).

[11] C. L. Evans, E. O. Potma, and X. S. Xie, Opt. Lett. 29, 2923 (2004).

[12] J.S. Bredfeldt, C. Vinegoni, D. L. Marks, and S. A. Boppart, Opt. Lett. 30, 495 (2005).

[13] M. Jurna, J. P. Korterik, C. Otto, J. L. Herek, and H. L. Offerhaus, Phys. Rev. Lett. 103, 043905 (2009).

[14] M. Jurna, J. P. Korterik, C. Otto, and H. L. Offerhaus, Opt. Express 15, 15207 (2007).

[15] U. Schnars and W. P. O. Juptner, Meas. Sci. Technol. 13, R85 (2002).

[16] J. Goodman, Introduction to Fourier Optics (Roberts \& Company Publishers, Greenwood Village, 2004).

[17] These values were obtained by spontaneous Raman measurements.

[18] Y. R. Shen, The Principles of Nonlinear Optics (WileyInterscience, New York, USA, 1984).

[19] E. Nichelatti and G. Pozzi, Appl. Opt. 37, 9 (1998).

[20] J. Vanroey, J. Vanderdonk, and P. E. Lagasse, J. Opt. Soc. Am. 71, 803 (1981). 